\section{Marking time}

\section{Beresford Hutchinson}

Revolution in Time: Clocks and the

Making of the Modern World.

By David S. Landes.

Harvard University Press: 1984. Pp.482. $\$ 20, £ 17$.

AN antiquarian book dealer, speaking of his life's passion which was not only his hobby but also his source of income, once said that book-collecting constituted a disease for which there is no known cure. Professor Landes would admit that lovers of old timepieces suffer from a similar affliction and that the horological version of the disease has long had him in its grip. Fortunately for historians and horologists, the general course of the illness and the patient's symptoms in his case have developed upon somewhat unusual lines. They have caused him to write a book - a book, moreover, which demonstrates not only his enthusiasm for mechanisms but, more especially, his fascination in the civil, scientific and economic reasons which engendered the invention of the mechanical clock, influenced its evolution, and gave rise to the variety of regions in which it was developed.

The period spanned in this book is roughly nine hundred years, beginning with Su Sung's Chinese water-clock, completed by AD 1094, and ending with the advent of the quartz-controlled timekeepers which have effectively brought the story of mechanical timepieces to its conclusion. The account embraces all the civilizations and countries which have made a contribution to horology, and describes the aspirations and frustrations of the protagonists in the story, together with the reactions which they generated.

From the formidable compilation of notes on the main text (78 pages worth), it is evident that the author has not merely relied on the standard horological literature. In this, he has followed the excellent example set by one of his mentors, Carlo Cipolla, another economic historian whose Clocks and Culture: 1300-1700 (Collins, 1967) represents an earlier attempt to deviate from the "How to do it - Who made it - How much is it worth" schools of horological authorship. It is perhaps a pity that Landes, unlike Cipolla, fails to provide a bibliography. Although all the relevant titles occur in the notes at the end of the book, a full reference, once given, is not repeated, and there are occasions when a brief reference precedes the full one. Fortunately, the comprehensive index usually provides the answer, but this entails a certain amount of to-ing and fro-ing which is vexing to the reader caught up in following the main thread of argument.

A historian with a practical bent, Professor Landes knows how clocks and watches are made and how they work, and a technical appendix offers the reader a clear explanation of the various forms of escapement - that part of a watch or clock which is the beating heart of all mechanical timekeepers. But this is really subordinate to his greater purpose, which is to consider the role of the mechanical clock and its influence on society.

In the first of three main parts, entitled "Finding Time", the author poses the questions, "Why clocks?", "Who needs them?", and concludes that, in mediaeval China, it was the astronomers who were largely to blame. In fourteenth-century Europe, there was an equally strong desire to make self-acting mechanical models of the Universe. Professor Landes is not convinced that these complicated astronomical machines gave rise to more simple clocks in their turn, but is willing to concede that the theory exists before dismissing it. "Keeping Time" and "Making Time", the other sections, respectively develop the themes of technical improvement and increased accuracy, and of methods of manufacture. The human element, whether applying to the makers or to the users, is always considered, with the result that this treatment of a highly technical subject can be understood and appreciated by a nontechnical reader.
Another of Professor Landes's purposes is to analyse the reasons for the rise of a horological industry in one country, often at the expense of another's. Why was the English watchmaking trade in such a decline by the end of the nineteenth century? Were the Swiss really caught napping by the burgeoning Japanese electronics industry in the early 1970s? Was the late nineteenth century machine-made American watch ever a serious rival to the high-grade Swiss and English hand-made product? At the risk of spoiling one story by giving away its ending, I shall say that, yes, the Americans had the Swiss running-scared for a brief period, although the Swiss made a brilliant recovery, partly by exporting a series of "look-alike" watches which had the American public fooled, but mostly by emulating American production methods.

Professor Landes is a humorous author as well as a questioning, impartial one. If he lapses into the occasional colloquialism, it adds to the enjoyment of his book rather than detracts from it. The section headings - "The Man Who Stayed to Dinner" (answer, John Harrison) and "Who Killed Cock Robin" (pass) - are examples of well-chosen titles which refer to section contents. His impartiality stems from wide reading and a total grasp of his subject, though this is to accuse him neither of sitting on the fence, nor of producing a "scissors-and-paste" book.

Are there any gaps in the presentation? Landes might have laid more stress on the fact that the precision timekeepers used at sea and in observatories led to a general raising of standards in domestic clockwork, especially in the late eighteenth century. More recently, what about the electric synchronous clock, which was heralded as bringing accurate time from the national grids into everybody's home from the 1930 s onwards? No mention of those, as far as I can see, and never mind at that: I would not have one in the house, any more than I should care to wear one of the electronic miracles on my wrist.

It is obvious that even horologists' passions can be aroused, and there are doubtless some passages in Professor Landes's book which will provoke discussion in other circles. In the meantime (no pun intended, as Landes says in his account of Greenwich), read his book and, preferably, purchase your own copy. Failing that, look out for it in the reference library under the general series 681 , and keep on pestering your librarian until it appears.

Art of the clock-maker - a French striking clock in the form of a suspended terrestrial globe, by Jacques de la Garde, Blois, 1552 . Originally it would have been fixed in the centre of an armillary sphere.
Beresford Hutchinson is Curator of Horology at the Old Royal Observatory (National Maritime Museum), Greenwich, London. 